\title{
Performance enhancement of wireless mobile adhoc networks through improved error correction and $\mathrm{ICl}$ cancellation
}

\author{
Zeeshan Sabir", M Inayatullah Babar and Syed Waqar Shah
}

\begin{abstract}
Mobile adhoc network (MANET) refers to an arrangement of wireless mobile nodes that have the tendency of dynamically and freely self-organizing into temporary and arbitrary network topologies. Orthogonal frequency division multiplexing (OFDM) is the foremost choice for MANET system designers at the Physical Layer due to its inherent property of high data rate transmission that corresponds to its lofty spectrum efficiency. The downside of OFDM includes its sensitivity to synchronization errors (frequency offsets and symbol time). Most of the present day techniques employing OFDM for data transmission support mobility as one of the primary features. This mobility causes small frequency offsets due to the production of Doppler frequencies. It results in intercarrier interference (ICI) which degrades the signal quality due to a crosstalk between the subcarriers of OFDM symbol. An efficient frequency-domain block-type pilot-assisted $\mathrm{ICI}$ mitigation scheme is proposed in this article which nullifies the effect of channel frequency offsets from the received OFDM symbols. Second problem addressed in this article is the noise effect induced by different sources into the received symbol increasing its bit error rate and making it unsuitable for many applications. Forward-error-correcting turbo codes have been employed into the proposed model which adds redundant bits into the system which are later used for error detection and correction purpose. At the receiver end, maximum a posteriori (MAP) decoding algorithm is implemented using two component MAP decoders. These decoders tend to exchange interleaved extrinsic soft information among each other in the form of log likelihood ratio improving the previous estimate regarding the decoded bit in each iteration.
\end{abstract}

Keywords: MANET, OFDM, Channel equalization, Intercarrier interference (ICI), PCCC encoder, MAP decoder, Channel estimation, Turbo codes

\section{Introduction}

Mobile adhoc network (MANET) represents a set of wireless mobile nodes that have the capability of communicating with each other without the help of a preexisting infrastructure [1]. Power consumption is an important issue in MANETs due to high dependence of mobile devices on battery power. A high data rate transmission technique like orthogonal frequency division multiplexing (OFDM) is ideal for MANETs at the Physical Layer due to its spectral efficiency. A number of applications like 802.16e (WiMax), 802.11a/g (WLAN), 802.11n (WiFi), etc., uses the multicarrier OFDM technique due to its bandwidth-efficient nature. OFDM is a very strong competitor for the Next Generation (4 G)

* Correspondence: zeeshansabir@nwfpuet.edu.pk

University of Engineering\& Technology, Peshawar, Pakistan
Wireless Communication techniques [2]. A peak data rate upto 54 Mbps can be supported by using commercial wireless local area network (WLAN) based on OFDM [3].

The subcarriers of OFDM system overlap in frequencydomain, and this property dictates its bandwidth efficiency. Figure 1a shows that orthogonality which is the nub of OFDM is maintained in the inverse fast Fourier transform (IFFT) block for the same OFDM symbol, by aligning peak of one subcarrier with the nulls of the others. Figure 1b shows an envelop of subcarriers which modulates in the IFFT block to form one OFDM symbol.

Intersymbol interference (ISI), which refers to the aliasing between OFDM symbols, is prevented by the introduction of a guard interval (GI) between the adjacent OFDM symbols. Length of the GI has a major 

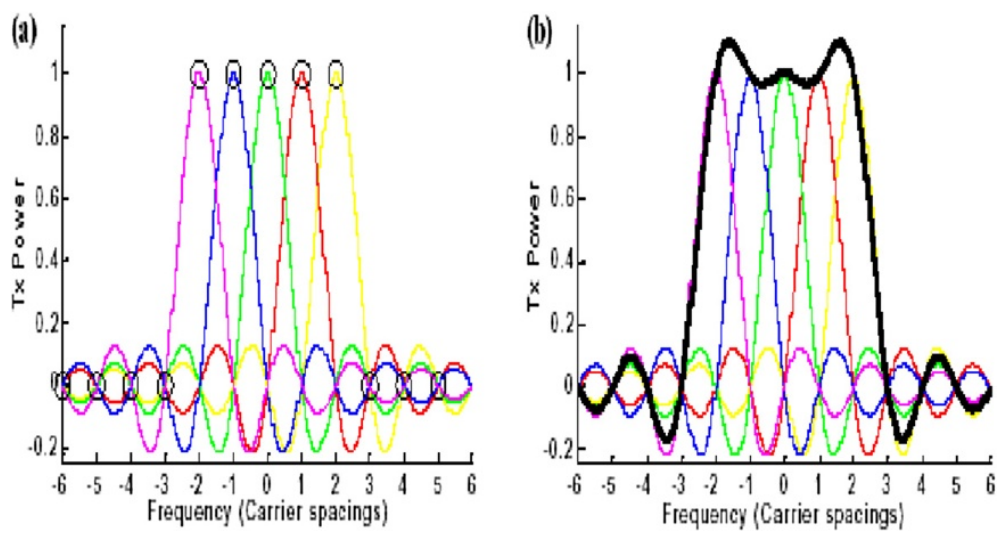

Figure 1 OFDM symbol comprising of multiple orthogonal subcarriers. (a) Perfect OFDM subcarriers. (b) An envelop of subcarriers.

impact on the ISI mitigation capability of the system. For mitigating the effects of ISI efficiently, the length of the GI should be chosen greater than the delay spread of the channel which refers to the time lapse at the receiver amid arrival of first and the last multipath components.

On the other side, major cause for intercarrier interference (ICI) is the Doppler frequency generated due to the relative motion between transmitter and receiver. Because of the relative mobility between transmitter and receiver, the received number of frequency cycles is different from the transmitted one. The difference in the number of frequency cycles tends to commove the alignment of peaks and nulls of subcarriers. This misalignment of peaks and nulls of subcarrier originate carrier frequency offsets that generate ICI in the system.

Turbo codes introduced in 1993 [4] first time gave the idea of channel codes that can possibly work in $0.5 \mathrm{~dB}$ range of the Shannon capacity limit. Turbo codes tend to add redundant information into the message bits that is later used at the time of decoding for improving the final estimate regarding the transmitted information. Two or more parallel concatenated recursive systematic convolutional (RSC) encoders constitute the basic structure of turbo codes. These RSC encoders are separated in parallel by an interleaver, that tends to spread any possible error, uniformly over all the generated bit stream, thus avoiding the infection of a single user or group of users from effect of the error. At the receiving side an iterative mechanism, based on maximum a posteriori (MAP) algorithm [5], is employed to decode the bits. We have incorporated turbo codes into the proposed OFDM model to palliate channel-induced noise effects from the transmitted OFDM symbols.

Various models have been proposed for OFDM concerning mitigation of ICI and error correction. A selfICI cancellation scheme has been purposed in [6] in which an idea of giving diverse weights to the same data symbol is proposed. ICI has notably been cancelled-out by modulating alike data symbol on the neighboring subcarriers and assigning dissimilar weights to them at the transmitter end. Maximal ratio combining algorithm is implemented at the receiver side to combine all these copies. In spite of well cancellation of ICI, high wastage of bandwidth dismisses the usage of this algorithm in the practical systems.

Jeon et al. [7] proposed an ICI cancellation model that is founded on the basic supposition that change in impulse response of the channel remains linear inside an OFDM symbol. Their proposed model was appropriate and applicable for low mobile environments only. It is because for the low mobile environments, channel exhibit a slow fading. Therefore, the impulse response variation for the same OFDM symbol can be considered linear. But for the high mobile environments, impulse response variations do not remain linear inside an OFDM symbol. It is because the channel follows a fast fading distribution. And thus we have to consider the fading occurring at each of the subcarrier independently irrespective of its neighboring subcarriers.

A multi-rate sampling theory is proposed by Chayratsami and Wickert [8]. This theory is applied at the receiving end and it focuses the extended outer spectrum of the OFDM subcarriers to decrease the interference. The interference between the remaining subcarriers of the OFDM is mitigated by applying a sequential interference cancellation (SIC) algorithm. A 10e-2 bit error rate (BER) performance is reported in the results at affordable SNR. The inherent latency and the high computational complexity involved in the processing of SIC algorithm dismiss the realistic implementation of the theory.

Li et al. [9] presented an adaptive minimum mean square error (MMSE) channel estimator. This estimator investigates the correlation of a dispersive channel frequency response over frequency-domain and time-domain. Again the computational complexity required for 
computing this correlation results in a notable delay which prevents the use of the proposed model for realtime services.

In this article, we propose a novel channel equalization technique based on frequency-domain block-type pilotassisted channel estimation (PACE) strategy that uses turbo detection-based approach at the receiver side. In the presented scheme, the information symbol detection phase is accomplished with the help of iterations of the error-correcting codes. We employed turbo codes in our presented OFDM model due to their improved error-correcting performance over the contemporary channel codes [10,11]. Turbo codes consist of parallel concatenation of RSC encoders. The number of parallel RSC encoders depends upon the coding rate that we want to achieve in our system. MAP algorithm-based decoder is used at the receiver side due to its better performance [12]. It decodes the received data bits using the iterations of the component MAP decoders. The two component MAP decoders exchanges a soft (a priori) information between each other during each iteration. This exchange of information leads to an improved estimate of the decoded bit as the iterations carry-on. Final estimate is made based upon the MAP probability calculated by the component MAP decoder no. 2. This results in a considerable performance enhancement of the system in each iteration by lowering of BER.

The rest of the article is organized as follows. "System model" section explains in detail the proposed system model along with its mathematical manipulations. "Algorithm for channel estimation and equalization" section incorporates the explanation of the channel estimation and equalization algorithm used in the presented model. "Anatomy of encoder and decoder" section contains the detail explanation of the structure of turbo encoder and MAP decoder used at the transmitter and receiver side, respectively. Simulation results have been discussed in "Simulation results" section and "Conclusions" section concludes the study.

\section{System model}

Figure 2 shows the proposed system model. First of all message bit sequence is passed through a source encoder which works as a compression block and converts the analogue message signal into binary digits with minimum redundancy. Any suitable source encoder, e.g., Huffman coder, can be used for performing this task. After this step the message bit sequence is passed through channel coding block for adding redundant bits for error detection and correction. In our case, we have used a rate $1 / 3$ turbo encoder. Structure of the turbo codes is discussed in "Anatomy of encoder and decoder" section in detail. After adding redundancy, the bits are digitally mapped and S/P converted. The digitally mapped symbols are then fed to the $N$-pt IFFT block, which produces $X_{p}(k)$ symbol at the output having $N$ subcarriers given by [13]

$$
X_{p}(k)=\left[X_{p}(0), X_{p}(1), X_{p}(2), \ldots, X_{p}(N-1)\right]^{T}
$$

IFFT block is responsible for generation of orthogonal subcarriers and modulation of digitally mapped data symbols on these subcarriers. For $n$ subcarriers $\left(V_{n}=0,1,2, \ldots, N-1\right)$ the OFDM-modulated symbol generated at the output of $N$-pt IFFT at time instant $p$ is given by

$$
X_{p}(k)=\frac{1}{N} \sum_{n=0}^{N-1} x(n) e^{\frac{-2 \pi j}{N}}
$$

where $X_{p}(k)$ is given by (1). $x(n)$ shows the signal at the output of the $n$th tap of IFFT block. This step is known as "OFDM modulation".

During this process, the pilot symbols are inserted in parallel and at the receiving end they are used for calculating $H$ matrix which consists of channel estimates for different subcarrier positions and is later used for data equalization. Next cyclic prefix, also called GI, is affixed with each OFDM symbol, i.e., last $L$ symbols of $X_{p}(k)$ are appended to the start of each transmitted OFDM block ${ }^{\sim} X_{p}$ shown as

$$
{ }^{\sim} X_{p}=X_{p}(k)\left(N-L_{c p}+1\right) ; X_{p}(k)
$$

where $L_{\mathrm{cp}}$ is the length of the cyclic prefix and $N$ represents the size of the IFFT block. Semicolon between the two terms shows that the first portion of the symbol is appended to the second symbol at the start. It is same as being done in MATLAB ${ }^{\circledR}$.

As we have discussed earlier in the first section, the necessary condition for the GI length for completely mitigating the effect of ISI is $D \leq L$, i.e., the GI length $L$ must be chosen such that its value should be at least equal to the channel delay spread.

In order to imitate the effect of real channel, Rayleigh fading additive white Gaussian noise (AWGN) channel has been simulated after passing through which, signal at the receiver side is given by

$$
y(n)=\sum_{l=0}^{L-1} h(n, l) x(n-l)+w(n)
$$

where $L$ represents the total number of discrete multiple paths for the Rayleigh fading channel, $h(n, l)$ shows the fading channel impulse response and $w(n)$ depicts the receiver added AWGN noise at instant $n$.

After removal of GI at the receiver, data is converted from serial to parallel. Next "OFDM demodulation" step is carried-out which involves passing the signal through FFT block. FFT block demodulates the data symbols 


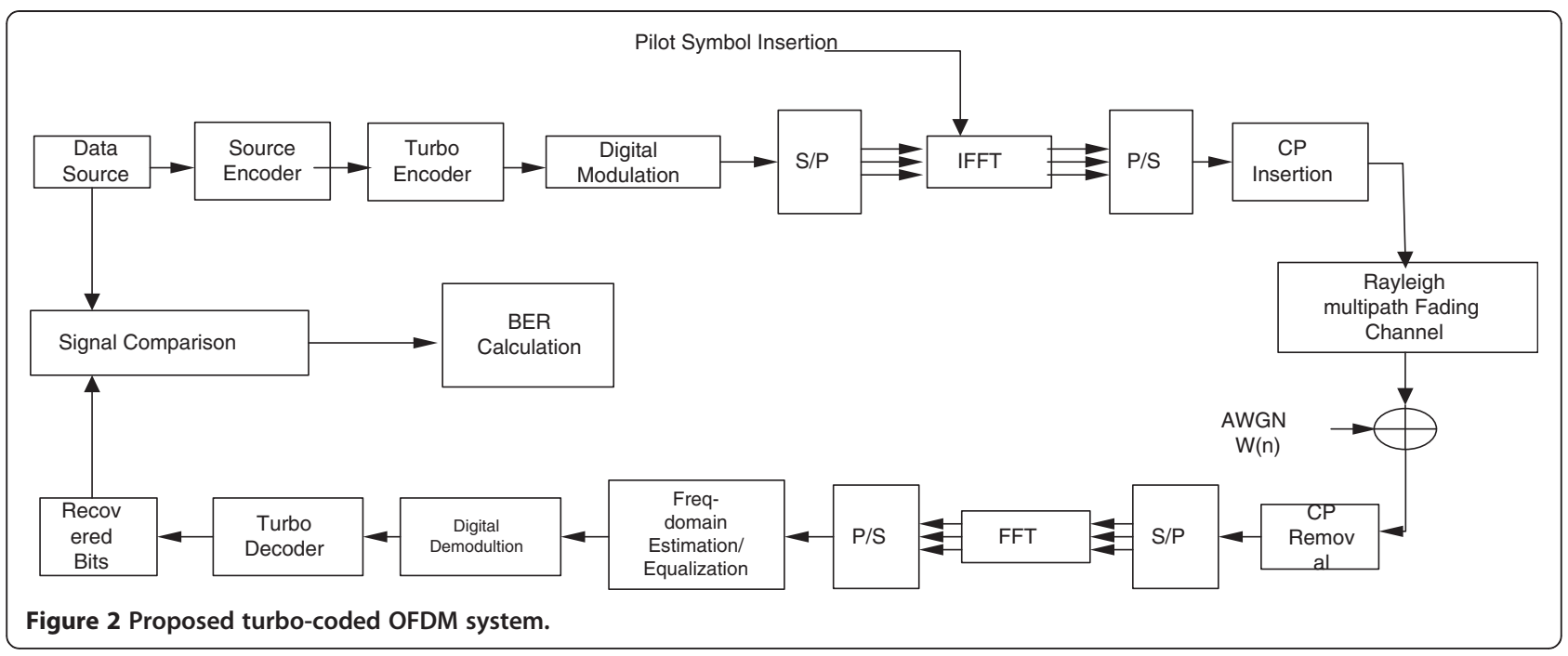

from the orthogonal subcarriers and the signal at the output of this block is given by [4]

$$
Y(m)=\sum_{k=0}^{N-1} \sum_{l=0}^{L-1} X_{k} H_{l}^{(m-k)} e^{\frac{-j 2 \pi k j}{N}}+W_{m}
$$

Where $W_{m}$ represents the FFT of the AWGN noise $W_{n}$ and $H_{l}^{(m-k)}$ represent the FFT of the Rayleigh fading channel impulse response given by

$$
H_{l}^{(m-k)}=\frac{1}{N} h_{n, l} e^{\frac{-j 2 \pi(m-k)}{N}}
$$

The data symbols after being demodulated from the orthogonal subcarriers are fed to the channel equalization/estimation block which uses pilot tones to remove the effects of impulse response offered by the channel from the received signal. As discussed in "Algorithm for channel estimation and equalization" section, efficient pilot tones' placement in an OFDM symbol plays an important role in the performance improvement of the channel estimator against the AWGN multipath Rayleigh fading channel. Finally, decoding of the bits is carried-out by passing them through iterative decoding portion of the receiver. The decoder is based on MAP algorithm which efficiently decodes the bits using two component MAP decoders by exchanging soft information between them. This step helps improve the decoding efficiency by using the previous estimates of the decoded bit in each iteration. In order to compute the BER performance of the system, the decoded signal is then passed through a signal comparator which takes as input the original user data bits and the decoded data bits. Finally, BER is computed using these two values in the signal comparator. The proposed OFDM model has been tested by changing the number of iterations of MAP decoder and evaluating and comparing its performance under four different digital modulation schemes.

\section{Algorithm for channel estimation and equalization}

The channel estimation and equalization technique implemented in the proposed system uses PACE approach. In this method, pilot symbols send prior to every data block nullify the impulse response effects offered by the channel from the received data symbols. These pilot symbols use to embed the effect of the channel impairments in them. Equalization technique implemented at the receiver uses these channel impairments to compensate the effects of the impulse response offered by the channel from the received user data. Pilot tones' placement in the symbol cast a great impact on the channel estimator performance. In our proposed model, we have used pilot-assisted block-type single-dimensional frequency-domain channel estimation strategy [14] as shown in Figure 3a. The system overhead for channel estimation was calculated as $14.3 \%$.

Instead of evenly spreading the pilot tones over all the transmitted data block such that some of the subcarriers in each data block are dedicated for sending the pilot data, as being done in comb-type estimator (Figure 3b), we used block-type estimation approach where some of the symbols are dedicated for sending pilot data while the user data are carried on rest of the symbols. Block-type channel estimator is the closest competitor of comb-type estimator, but the problem in comb-type pilot-inserted channel estimator is that it uses interpolation for calculating the channel impulse response at those subcarrier positions where pilot tones are not present. This introduces an interpolation error that adds to the overall BER 


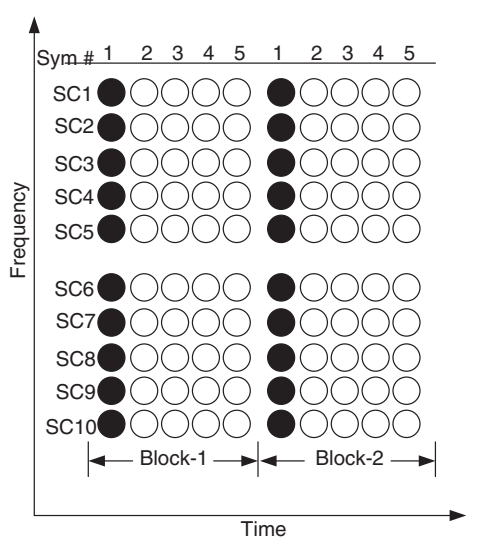

(a) Block-type pilot assisted CE

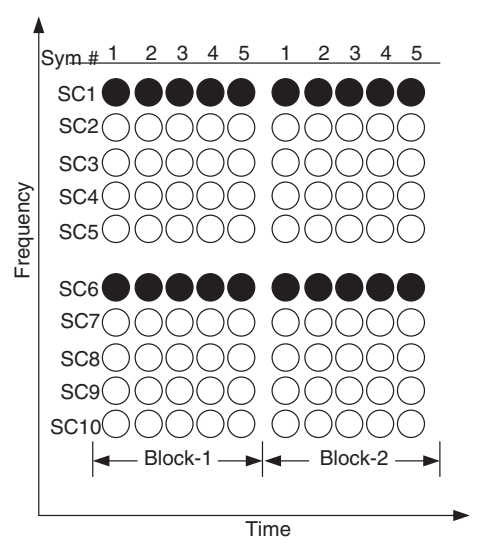

(b)Comb-type pilot assisted CE

\section{SC=Sub Carrier \# , Sym=OFDM symbol \# , \\ =Pilot data, $\bigcirc=$ Information data}

Figure 3 Schematic representation of block-type and comb-type pilot tone insertion techniques.

performance of the model. Channel estimator overhead was same in our case compared to comb-type technique. A schematic comparison of both types of the channel estimators is given in Figure 3 [14].

The upcoming data symbols have been equalized using the cannel taps calculated from the pilot OFDM symbol. This process is repeated on symbol-to-symbol basis until next pilot OFDM symbol arrives in the next data block as shown in Figure 3 above. Figure 4 shows the block diagram for the proposed channel estimator.

As per procedure, computation of $H_{\mathrm{ce}}$, the channel estimation matrix is carried-out with the help of received and sent pilot symbols using the relation:

$$
H_{c e}=\frac{Y_{p}(k)}{X_{p}(k)} \quad k=0,1,2, \ldots, N_{p}-1
$$

Where $X_{p}(k)$ and $Y_{p}(k)$ represent, respectively, the transmitted and received pilot tones.

The upcoming data equalization is performed by multiplying the inverse of the channel estimation matrix obtained in the first step with the received data symbol.

$$
X_{e}(k)=Y(k) H_{c e}^{-1} \quad k=0,1,2, \ldots, N-1
$$

If the delay elements offer a time delay of $t$ then the equalizer's frequency response $H_{\text {eq }}(f)$ is periodic with a period of symbol rate while the symbol rate equals $1 / t$. This shows that it satisfies the Nyquist's first criterion which states that for achieving a zero ICI with perfect equalization, the pulse shape should be such that it has a non-zero amplitude at its centre $(t=0)$ and zero amplitudes at $t= \pm n T\left(V_{n}=1,2,3, \ldots\right)$. Thus, in order to optimally attain the equalization state, the following condition is mandatory to be followed by the equalizer and frequency response matrix of the channel:

$$
H_{c h}(f) H_{e q}(f)=1:|f|<\frac{1}{2} t
$$

Here $H_{\mathrm{ch}}(f)$ represents the folded frequency response of the channel. Thus, the zero forcing equalizer has been implemented as an inverse filter that equalizes the

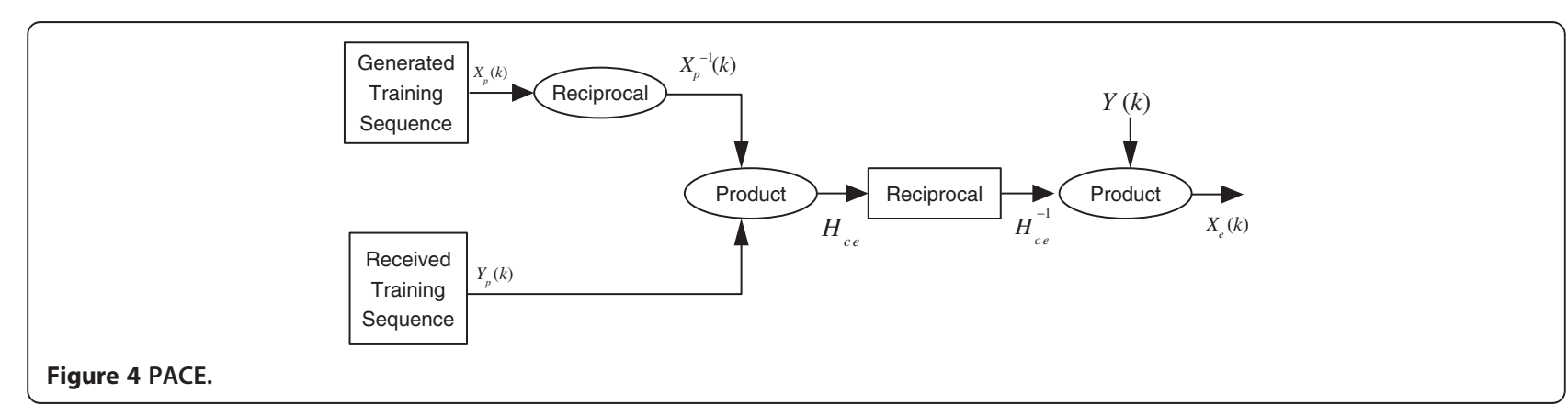




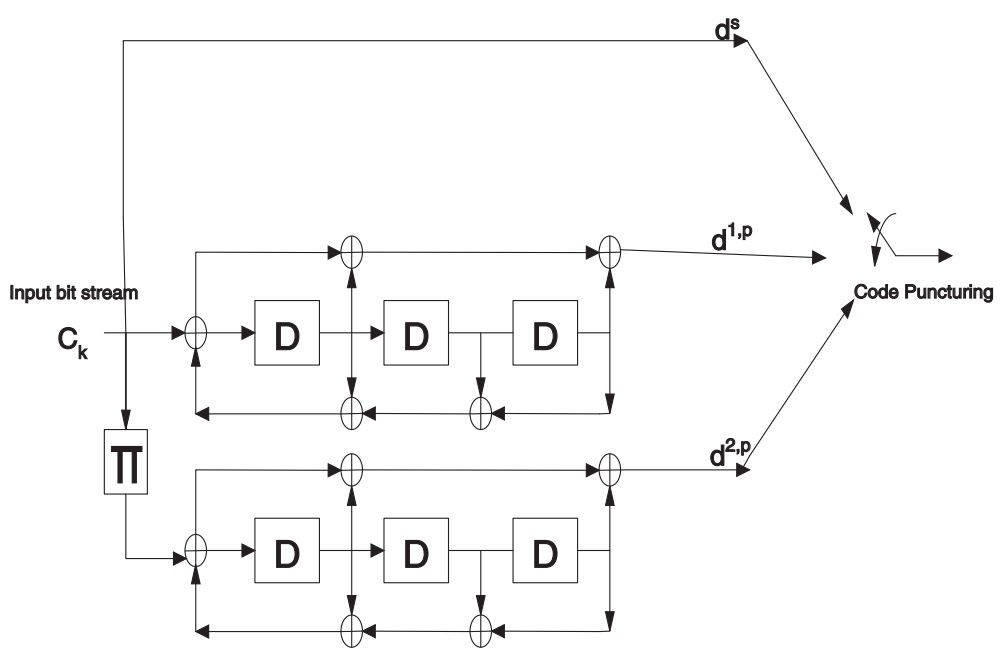

Figure 5 A rate $1 / 3$ PCCC turbo code.

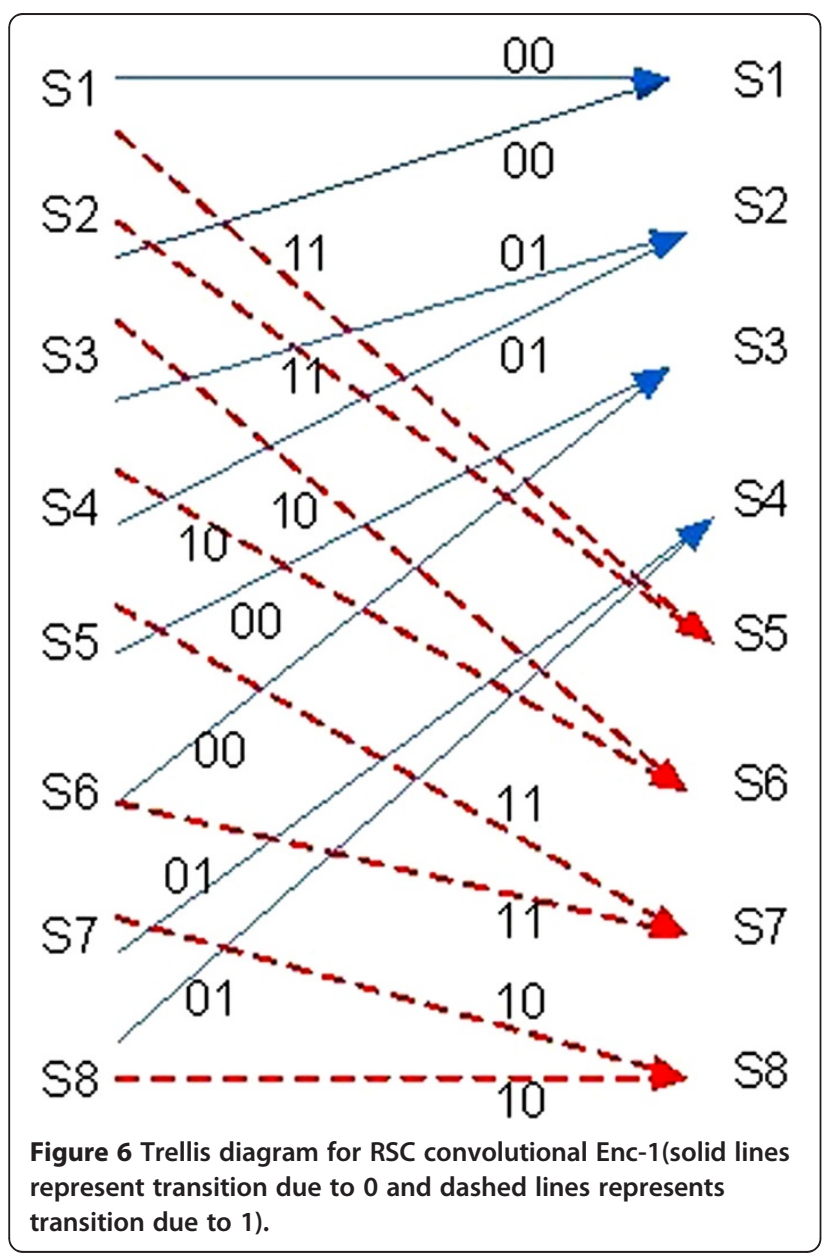

received data symbols impulse response by inverting the folded frequency response of the channel.

The above process is repeated for each data symbol till the effect of ICI is equalized for the whole received sequence.

\section{Anatomy of encoder and decoder Encoder}

We used a $1 / 3$ rate turbo encoder in the suggested OFDM system. The encoder works on the principal of adding redundant information into the source-coded message bits for minimizing the transmission errors. Figure 5 shows the proposed encoder structure. It is made by concatenating in parallel two RSC encoders and using an interleaver in between.

In order to variate the coding rate as per requirement, puncturing is used at the output. After puncturing, the output bit sequence can be represented as

$$
S_{1} P_{1}^{1} P_{1}^{2} S_{2} P_{2}^{1} P_{2}^{2} S_{3} P_{3}^{1} P_{3}^{2} \ldots S_{n} P_{1}^{n} P_{2}^{n}
$$

Table 1 The eight possible states for constraint length 3 RSC convolutional encoder

\begin{tabular}{llll}
\hline State & 1st Delay reg & 2nd Delay reg & 3rd Delay reg \\
\hline S1 & 0 & 0 & 0 \\
S2 & 0 & 0 & 1 \\
S3 & 0 & 1 & 0 \\
S4 & 0 & 1 & 1 \\
S5 & 1 & 0 & 0 \\
S6 & 1 & 0 & 1 \\
S7 & 1 & 1 & 0 \\
S8 & 1 & 1 & 1 \\
\hline
\end{tabular}




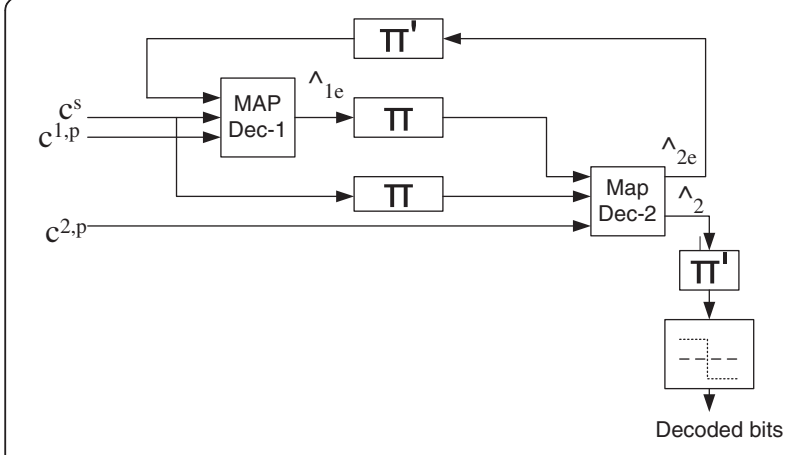

Figure 7 Turbo MAP decoder based on two component decoders.

where the superscript corresponds to the component RSC convolutional encoder number and the subscript represents the bit number for that component encoder.

The outputs $d^{1, p}$ and $d^{2, p}$ represent the parity bits contributed by the first and second RSC encoders while $d^{s}$ corresponds to the systematic bits. The generator sequence of the component encoders plays a vital role in the system's performance. The feed-forward and feedbackward generator sequences used in our model are $1+$ $D+D^{2}+D^{3}$ and $1+D+D^{3}[15]$, respectively.

Simplified trellis diagram of the RSC convolutional encoder is mentioned in Figure 6. Whereas Table 1 shows the eight possible states for the constraint length 3 RSC convolutional encoder. The output code word generated by each transition is shown with the transition arrow.

\section{Decoder}

Decoder is based on MAP decoding algorithm. Two serially concatenated component MAP decoders exchanges a priori soft information via an interleaver as shown in Figure 7 [16].
The exchange of soft information among the two component MAP decoders is done in an iterative manner. Dec-1 calculates likelihood ratio (LLR) as its soft information and this information regarding the decoded bits is fed as a priori probability to Dec- 2 .

$$
\operatorname{LLR}\left(u_{k}\right)=1 n\left(\frac{P\left(u_{k}=+1 / y_{1}^{N}\right)}{P\left(u_{k}=-1 / y_{1}^{N}\right)}\right)
$$

In the first iteration, as a starting value, for Dec-1 the a priori probability for 1 and 0 bits is taken as $1 / 2$. For the uniform spread of transmission errors, the output of Dec-1 is interleaved and thus produces a better estimate for information sequence of Dec-2. The interleaver provides scrambled information for the second component MAP decoder so that the inputs of the two component MAP decoders should be decorrelated and a suboptimal decoding algorithm based on the decorrelated information exchange can be applied among the component MAP decoders. The a posteriori probability produced by Dec-2 acts as the extrinsic information of the decoded bits for Dec-1 and is fed, via a deinterleaver, to it. Thus, as the iterations carry-on, the final estimate of the decoder regarding the input bit keeps on improving compared to a one iteration hard decision-based serially concatenated decoder. As iterations carry-on, the two component decoders' output which is in the form of their extrinsic information begins to diverge from its starting value of $1 / 2$. After executing $k$ iterations, the output of component MAP decoder \# 2 is passed through a hard decision. The value of $k$ is fixed by the system designer depending upon the trade-off between the error sensitivity of the system for the application for which it is designed for and the latency, dictated by the computational complexity, tolerable by the system. Non-realtime services are error sensitive but are delay tolerant while real-time services are delay sensitive though error tolerant. Thus, this trade-off is application dependent.

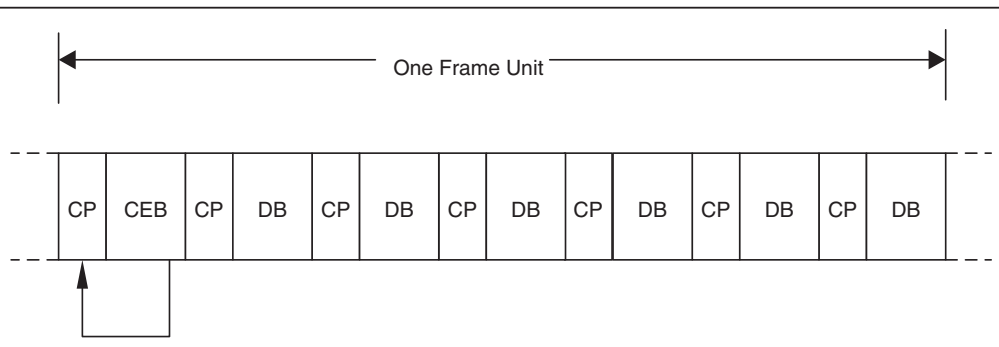

$\mathrm{CP}=$ Cyclic Prefix, $\mathrm{CEB}=$ Channel Estimation Block, DB=Data Block

1 Frame Unit=1 CE Block+ 6 Data Blocks[17]

Figure 8 Frame structure simulated for data transmission of the proposed model. 
For a rate $1 / n$ component codeword, first MAP decoder produces the LLR given by [14]

$$
\Lambda_{1}\left(C_{t}\right)=\log \frac{\sum_{l^{\prime}, l=0}^{M_{s}-1} \alpha_{t-1}\left(l^{\prime}\right) p_{t}^{1}(1) \exp \left(-\frac{\sum_{j=0}^{n-1}\left(r_{t, j}-x_{t, j}^{1}(l)\right)^{2}}{2 \sigma^{2}}\right) \beta_{t}(l)}{\sum_{l^{\prime}, l=0}^{M_{s}-1} \alpha_{t-1}\left(l^{\prime}\right) p_{t}^{1}(0) \exp \left(-\frac{\sum_{j=0}^{n-1}\left(r_{t, j}-x_{t, j}^{0}(l)\right)^{2}}{2 \sigma^{2}}\right) \beta_{t}(l)}
$$

where $p_{t}^{1}(0)$ and $p_{t}^{1}(1)$ are a priori probability at the input of the component MAP Dec- 1 for bit 0 and 1 , respectively, and is taken as 0.5 in the first iteration. Similarly, $p_{t}^{2}(0)$ and $p_{t}^{2}(1)$ represent a priori probability at the input for MAP Dec-2. The bit is decoded as a 1 or 0 depending upon the sign of the LLR, in the final iteration. After $k$ iterations, if LLR is positive, bit 1 is decoded and if LLR is negative, a 0 is decoded. These decoding of the bit 1 or 0 corresponds to the inherent property of the log operator which dictates a positive mantissa whenever the numerator term of the log is greater than denominator and negative mantissa for the vice-versa case.

\section{Simulation results}

Using MATLAB ${ }^{\circledR}$ simulation, we analyzed the results of the introduced model with uncoded and turbo-coded OFDM with the aid of frequency-domain block-type PACE.
Figure 8 shows the frame structure that was simulated for the transmission of OFDM symbols. Rayleigh slow fading frequency-selective AWGN channel has been simulated to analyze the performance of the proposed model.

\section{Test case 1. Uncoded OFDM with PACE}

In the proposed model, OFDM was used with a symbol size of 256 subcarriers and ISI effects were vanished with a guard interval length of 64 subcarriers.

As mentioned in Figure 8, every frame unit of OFDM consists of one channel estimation pilot symbol followed by six data symbols. Data rate of the transmission was calculated as $69.37,46.25,23.15$, and $11.57 \mathrm{MHz}$, respectively, for 64-Quadrature Amplitude Modulation (QAM), 16-QAM, Quadrature Phase Shift Keying (QPSK), and Binary Phase Shift Keying (BPSK). 17.4-MHz bandwidth was used to conform with $802.11 \mathrm{n}$ standard.

Figure 9 shows degradation in the performance of the system as we move towards higher modulation schemes. The performance degradation is because of close positioning of signal points on the constellation map for higher modulation schemes as shown in Figure 10. The four panels in Figure 10 show the condition of the signal before and after its passage through the simulated channel for the four different modulation schemes mentioned below each of the panel. In each of the four panels, left graph shows the scatter plot of quadrature over in phase for transmitted signal $X$ while on the right graph we see the same plot for received signal $Y$.

We used PACE strategy with 1:6 pilot to data symbol ratio in the proposed model.

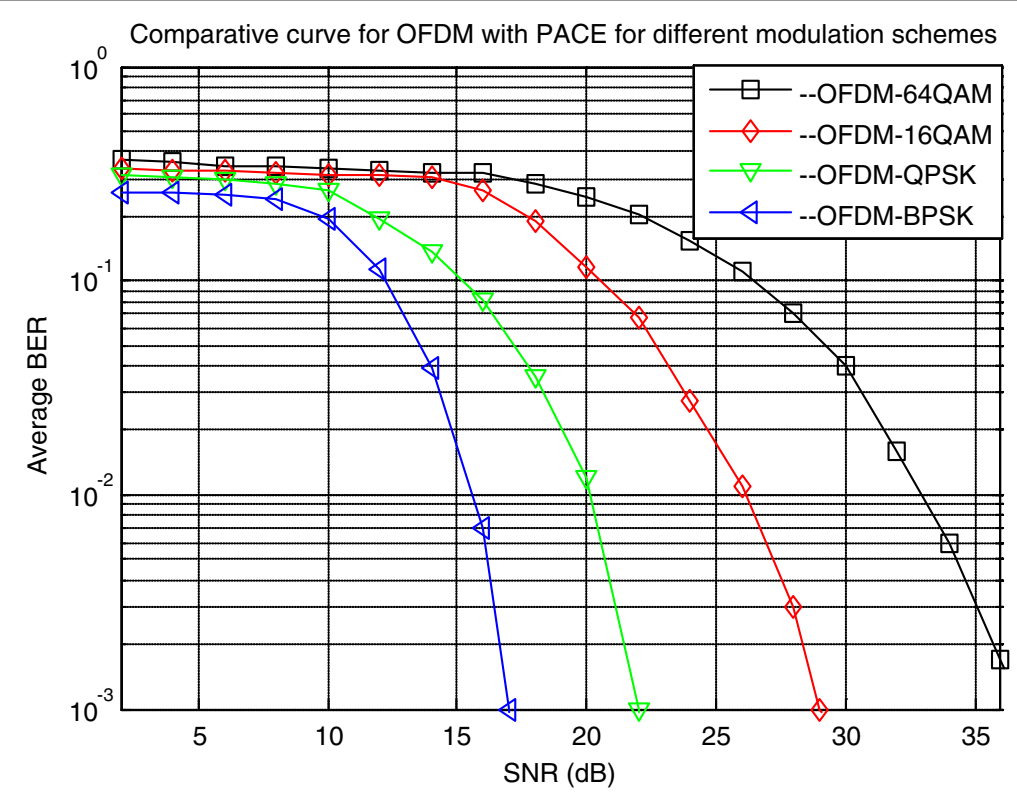

Figure 9 Uncoded OFDM with PACE through rayleigh fading channel. 

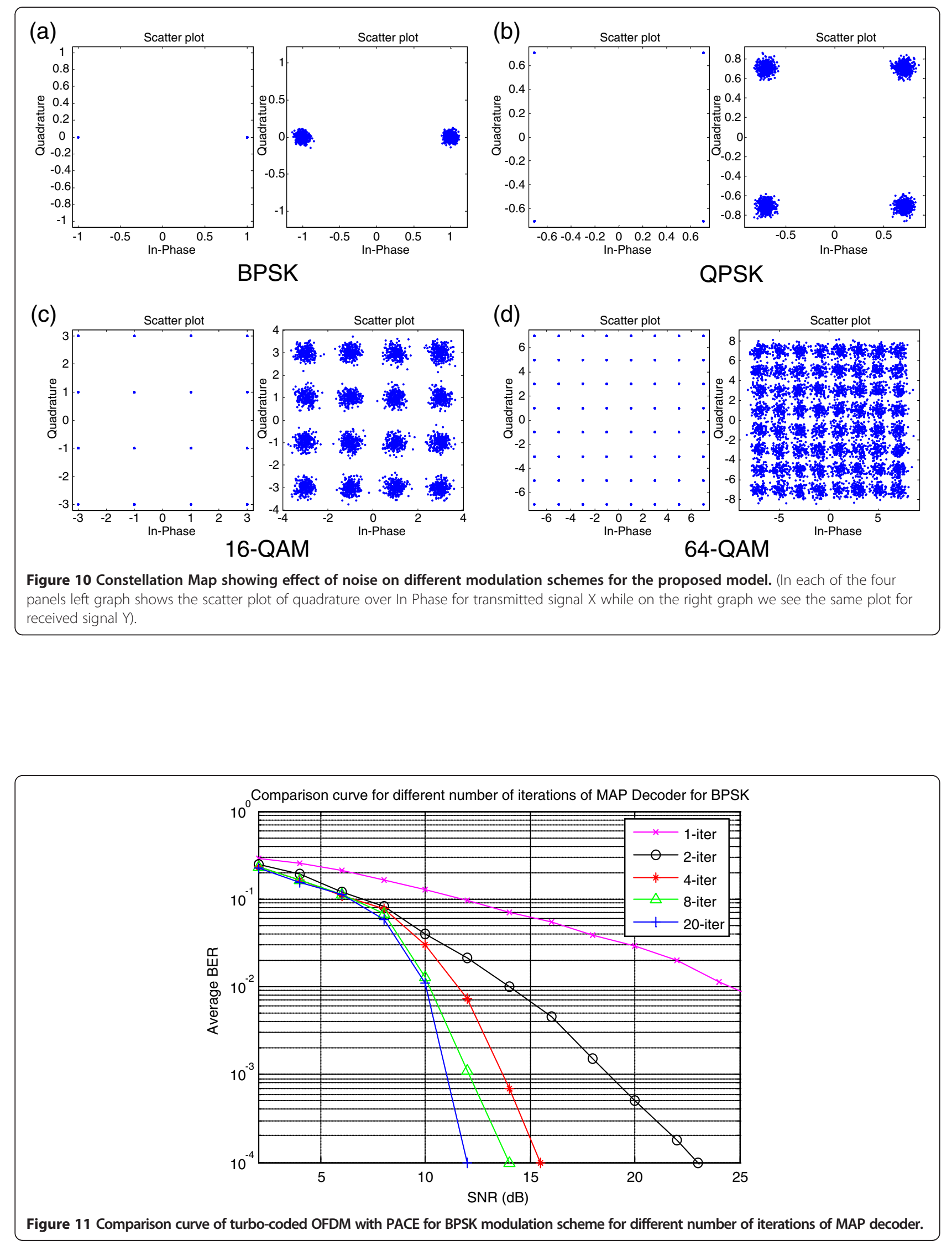


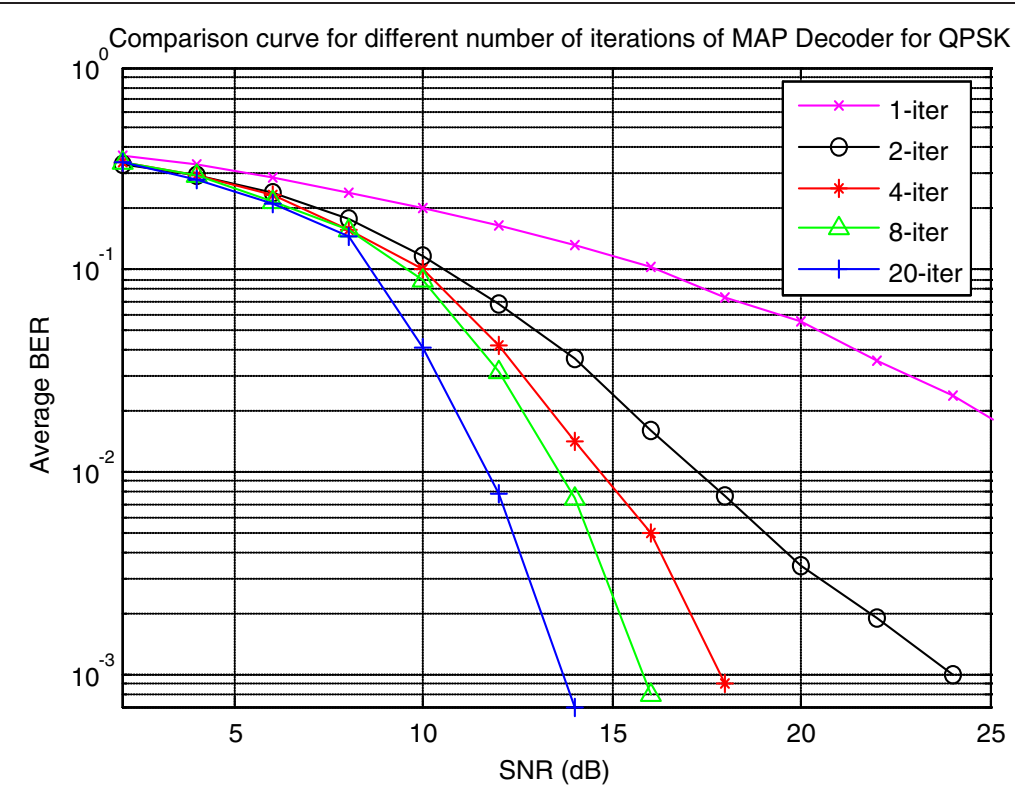

Figure 12 Comparison curve of turbo-coded OFDM with PACE for QPSK modulation scheme for different number of iterations of MAP decoder.

\section{Test case 2. Turbo-coded OFDM with PACE}

In the Test Case- 2 turbo codes were implemented in the same proposed model of OFDM with PACE. In this section, the system's behavior has been studied by changing number of iterations for the MAP decoder for different modulation schemes. The same multipath rayleigh fading AWGN channel model was used for these results. Number of iterations cast a major impact on the overall system performance. The standard RFC for 802.16e
(WiMAX) has fixed seven iterations for the MAP decoder. We have shown results in the section for $20,8,4$, 2 , and 1 number of iterations. The system shows a general trend of improvement in the performance as the iterations of MAP decoder are increased.

We have simulated the system for four different digital modulations: BPSK, QPSK, 16-QAM, and 64-QAM. OFDM symbol comprises of 256 subcarriers with GI length of 64 .

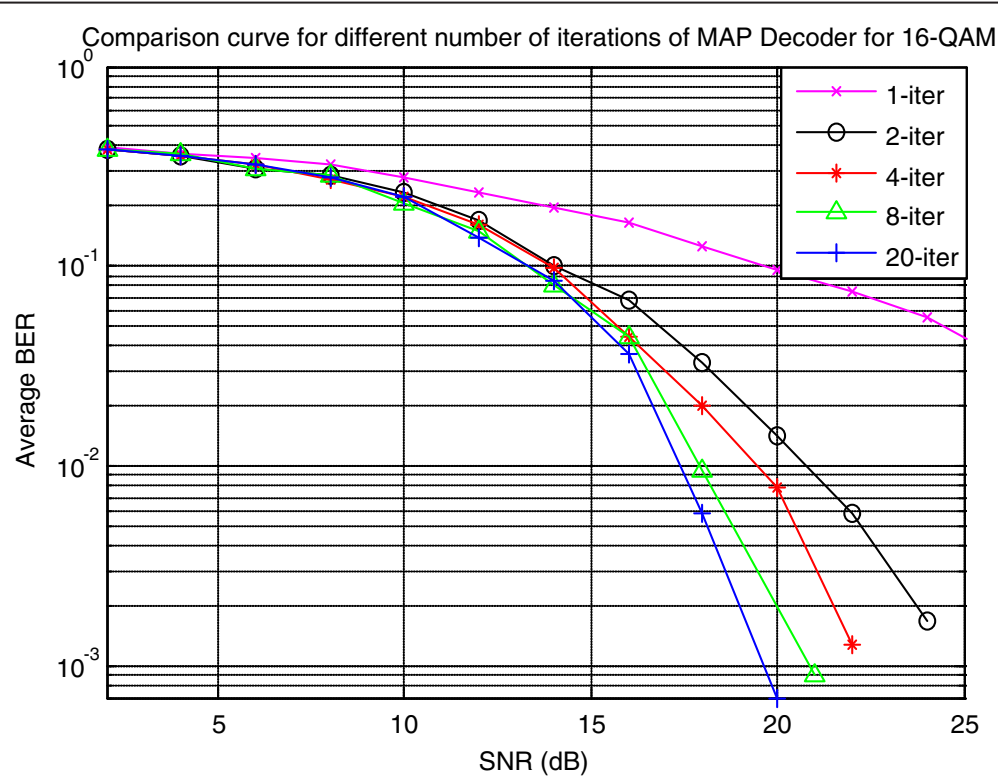

Figure 13 Comparison curve of turbo-coded OFDM with PACE for 16-QAM modulation scheme for different number of iterations of MAP decoder. 


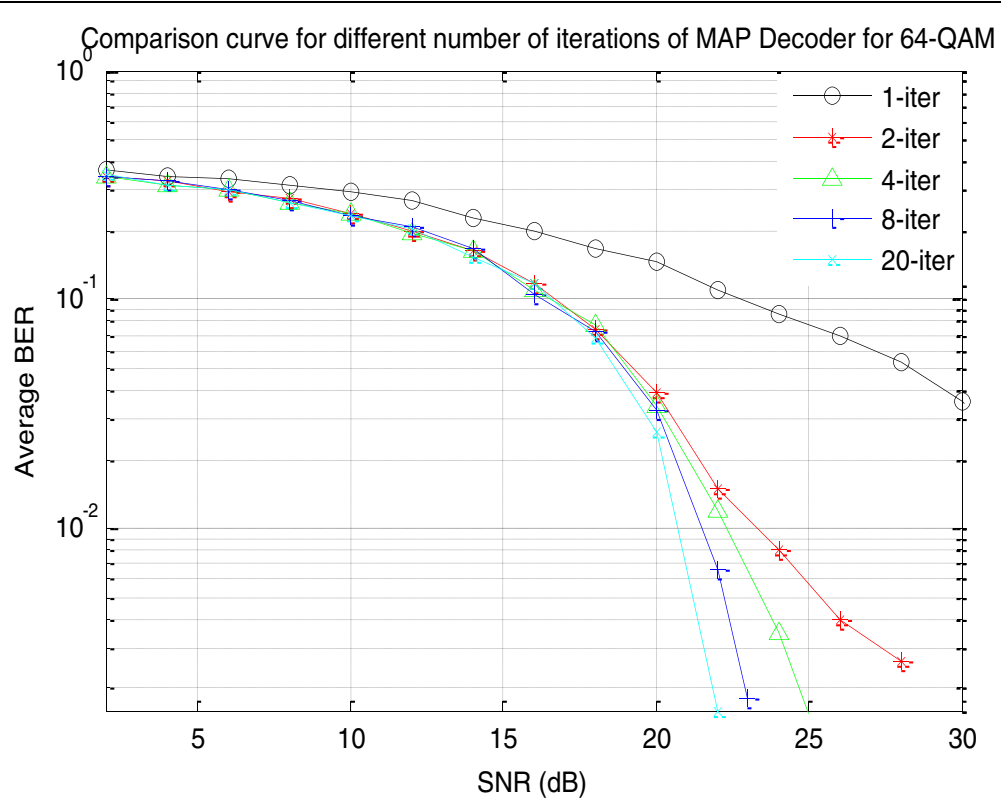

Figure 14 Comparison curve of turbo-coded OFDM with PACE for 64-QAM modulation scheme for different number of iterations of MAP decoder.

When compared with the curve given in Figure 9, BPSK shows a performance improvement of $4.9 \mathrm{~dB}$ for 20 iterations curve in Figure 11, which goes to the credit of using turbo codes in the proposed model. As discussed earlier, MAP decoding algorithm improves the system's performance by exchanging a soft decision among the two component map decoders. This performance improvement is depicted by a lowering of BER in these curves.

Similarly for QPSK, curves follow the same trend with the addition that performance improvement is more when compared with the curves in Figure 9. This is due to the fact that QPSK gains more from the application of turbo codes, compared to BPSK, because of the compact positioning of signal points in constellation diagram as shown already in Figure 10. For four iterations, the QPSK curve given in Figure 12 shows a performance improvement of $3.1 \mathrm{~dB}$ at 10e-3 BER performance when compared with the study of [18]. This improvement is due to the efficient pilot tone insertion technique employed in our system that removes the possibility of interpolation error in the decoded bit as estimation of the channel is carried out at all subcarrier positions of OFDM symbol.

The comb-type PACE technique used in [18] has an inherent interpolation error at the positions which are missing with the pilot tones. It is because in the frequencydomain pilot-assisted block-type channel estimation strategy, some OFDM symbols of all the frames are dedicated for sending pilot data. All the subcarriers of these OFDM symbols carry pilot tones for estimating the channel impulse response. Contrary to it, in the frequency-domain pilot-assisted comb-type pilot insertion technique, some subcarriers of all the OFDM symbols carry pilot data. For rest of the subcarrier locations which are missing with the pilot tones, a suitable interpolation technique, e.g., linear, cubic, spline, etc., is employed which calculates channel impulse response at the positions which are missing with the pilot tones. Because of the interpolation, comb-type PACE suffers with an inherent interpolation error which has been depicted by a 3.1-dB performance degradation when compared with the block-type PACE tested in our proposed model with turbo-coded OFDM system.

In Figures 13 and 14, the same parameters have been applied, respectively, for 16-QAM and 64-QAM for measuring performance of the system. Again as we move towards the higher modulation schemes, there is a considerable gain in the performance improvement in the system. As discussed earlier, it refers to the compact positioning of signal points in the constellation diagram which increase the chances of transmission errors. In the lower modulation schemes, the constellation points are already not in close proximity of each other so there are fewer chances for these points to merge and thus data corruption is also less likely to occur. The redundant bit added due to the application of turbo codes into such system appears to be just an extra consumption of energy. That's why application to turbo codes is more significant for higher modulation schemes as compared to lower modulation schemes. Thus, there should be a compromise between the acceptable error rate for which the system is designed and the tolerable latency, for the choice of a particular modulation scheme, while implementing error correction into the system as all these parameters are directly linked with each other. 


\section{Conclusions}

In this article, we have proposed a novel error correction and ICI cancellation scheme for OFDM system. It mitigates the effect of ICI using the channel estimation matrix calculated using pilot tones. Performance of the system is further improved under Rayleigh fading AWGN channel environment using error correcting turbo codes which effectively improves BER of the system. Simulation results using different modulation schemes show that the given algorithm can significantly improve OFDM system's performance which is the physical layer implementation of MANETs.

\section{Competing interests}

The authors declare that they have no competing interests.

\section{Acknowledgment}

The authors are thankful to Higher Education Commission (HEC), Pakistan, which supported this study under the Program "HEC Indigenous Fellowships Scheme, Batch-IV". The authors are also grateful to the reviewers who made constructive comments and gave their expert opinion to further improve the presentation of this article.

Received: 4 February 2012 Accepted: 17 September 2012

Published: 9 October 2012

\section{References}

1. H Dong, ID Chakeres, A. Gersho, EM Belding-Royer, JD Gibson, Selective biterror checking at the MAC layer for voice over mobile ad hoc networks with IEEE 802.11, in In Proceedings of IEEE Wireless Communications and Networking Conference, 2nd edn. (Atlanta, GA, USA, 2004), pp. 1240-1245

2. M Zhao, ZShi, MC Reed, Iterative Turbo Channel Estimation for OFDM system over rapid dispersive fading channel. IEEE Transaction on Wireless Communication. 7(8), 3174 - 3184 (August 2008)

3. I Daniele Lo, R Marco, T Luigi Della, O Fabio, MIMO-OFDM physical layer real-time prototyping, in In Proceedings of IEEE Wireless Communications and Networking Conference (Las Vegas, USA, 2008), p. 18-23

4. C Berrou, A Glavieux, P Thitimajshima, Near Shannon limit error-correcting coding and decoding: turbo codes, in in Proceedings of IEEE International Conference on Communications, ed. by, vol. 2 (Geneva, May 1993), pp. 1064-1070

5. HR Sadjadpour, Maximum a posteriori decoding algorithm for turbo codes, in In Proc. of SPIE, 4045th edn. (Orlando, FL, 2000), pp. 73-83

6. Y. Zhao, SG Haggman, Intercarrier interference self-cancellation scheme for OFDM mobile communication system. IEEE Trans. Commun. 49(7), 1185-1191 (2001)

7. WG Jeon, KH Chang, YS Cho, An equalization technique for orthogonal frequency-division multiplexing systems in time-variant multipath channels. IEEE Trans. Commun. 47(1), 27-32 (1999)

8. P Chayratsami, MA Wickert, Channel estimation and mitigation techniques for OFDM in a Doppler spread channel, in In Proceedings of IEEE Global Communications Conference, ed. by (New Orleans, LA, USA, 2008), pp. 1-5

9. $Y \mathrm{Li}, \sqcup$ Cimini, NR Sollenberger, Robust channel estimation for OFDM systems with rapid dispersive fading channels. IEEE Trans. Commun. 46(7), 902-915 (1998)

10. IA Chatzigeorgiou, MRD Rodrigues, IJ Wassell, RA Carrasco, A comparison of convolutional and turbo coding schemes for broadband FWA systems. IEEE Trans. Broadcast. 53(2), 494-503 (2007)

11. J Martins, A Giulietti, M Strum, Performance comparison of convolutional and block turbo codes for WLAN applications, in In Proceedings of 4thIEEE International Conference on Devices, ed. by (Circuits and Systems, Aruba, 2002), pp. 1-5

12. J Ortín, P Gracia, F Gutiérrez, A Valdovinos, Performance analysis of turbo decoding algorithms in wireless OFDM systems. IEEE Trans. Consumer Electron. 55(3), 1149-1154 (2009)

13. S Zeeshan, Y Syed-Abdur-Rehman, BM Inayatullah, WM Arif, Improved joint ICI cancellation and error correction for OFDM system, in In Proceedings of 2011 International Conference on Electronic Commerce, Web Applications and Communications, ed. by, vol. 1, 143rd edn. (Springer, Berlin, 2011), pp. 1-11

14. S Zeeshan, WM Arif, BM Inayatullah-Babar, OFDM, Turbo Codes and Improved Channel Estimation-A Magical Combination (VDM Verilog Publishers, Germany, 2011). ISBN: 9783639326505
15. B Sergio, B Ezio, Principles of Digital Transmission: With Wireless Applications, 1st edn. (Springer, New York, 1999)

16. V Branka, Y Jinhong, C Turbo, Principles and Applications (Kluwer Academic Publishers, London, 2002)

17. P Ramjee, OFDM for Wireless Communication Systems (Universal personal communication series, 2004)

18. S Elnoubi, S Elbadawy, C Shaban, Performance of turbo-coded OFDM system with comb pilot channel estimation in Rayleigh fading channel, in In Proceedings of IEEE Military Communications Conference, ed. by (San Diego USA, 2008), pp. 1-6

doi:10.1186/1687-6180-2012-216

Cite this article as: Sabir et al:: Performance enhancement of wireless mobile adhoc networks through improved error correction and ICI cancellation. EURASIP Journal on Advances in Signal Processing 2012 2012:216.

\section{Submit your manuscript to a SpringerOpen ${ }^{\circ}$ journal and benefit from:}

- Convenient online submission

- Rigorous peer review

- Immediate publication on acceptance

- Open access: articles freely available online

- High visibility within the field

- Retaining the copyright to your article

Submit your next manuscript at $>$ springeropen.com 